\title{
Por que os oceanos? Por uma diplomacia estratégica brasileira para a biodiversidade em alto-mar
}

\author{
Why the oceans? For a Brazilian strategic diplomacy for \\ offshore biodiversity
}

Igor Magri de Queiroz'

\begin{abstract}
Resumo: a comunidade internacional decidiu agir para preencher as lacunas na regulamentação internacional sobre o uso sustentável da biodiversidade além da jurisdição nacional (BAJN). Esta é uma resposta para os desafios impostos pelo Antropoceno, seja pela deterioração desses recursos, ou pelo crescente aumento da presença humana em atividades relacionadas à BAJN. Assim, o objetivo deste estudo é compreender a dimensão da governança global da BAJN. A metodologia adotada é qualitativa, baseando-se numa pesquisa bibliográfica. Os resultados apontam para uma necessidade de apreender a governança desses recursos por uma lente da complexidade, em que as diversas forças regendo essa governança são consideradas, para se obter uma visão sistêmica. O Brasil ainda não construiu uma resposta estratégica para a BAJN, o que enfraquece sua capacidade de desenvolvimento sustentável baseado nos oceanos. Assim, oferece-se a diplomacia estratégica como ferramenta para a atuação brasileira com relação à BAJN.
\end{abstract}

Palavras-chave biodiversidade além da jurisdição nacional; diplomacia estratégica; governança global; oceanos.

\begin{abstract}
: the international community has decided to act to fill in the gaps in the regulation of the biodiversity beyond national jurisdiction (BBJN). This is a response to the challenges posed by the Anthropocene either by the deterioration of these resources or by the increasing human presence in activities related to the BBNJ. Thus, the aim of this study is to understand the dimension of the BBNJ in global governance. The methodology adopted is qualitative, based on a bibliographical research. The results point to a need to grasp the governance of these resources through the lens of complexity, in which the many forces guiding this governance are considered, generating a systemic view. Brazil has not yet built a strategic response to the BBNJ, which weakens its capacity for sustainable development based on the oceans. Thus, the strategic diplomacy framework is offered as a tool for building a brazilian strategic diplomacy for the BBNJ.

Keywords: biodiversity beyond national jurisdiction; global governance; oceans; strategic diplomacy.
\end{abstract}

\section{Introdução}

Por que os oceanos? Além de cobrirem a maior parte do Planeta Azul, os oceanos são efetivos reguladores da temperatura atmosférica, além de sumidouros de gás carbônico e fonte de alimentos. Porém, as taxas de extinção de espécies têm aumentado rapidamente e a acidificação dos oceanos impõe desafios a um ecossistema pouco conhecido (CEBALLOS et al., 2017; MILLENIUM..., 2005;

1 Instituto de Relações Internacionais - Universidade de Brasília (IREL/UnB). 
SÁNCHEZ-BAYO, WYCKHUYS, 2019; WWF, 2018; UNEP, 2019). Estes são apenas alguns exemplos do que a humanidade enfrenta: o Antropoceno, uma época em que a humanidade passa a fazer parte dos principais fatores das mudanças ambientais globais (BEAU, LARRÈRE, 2018; BARROS-PLATIAU et al., 2015; WORKING..., [s.d.]). É um presente desafiador e um futuro incerto, especialmente quando o assunto diz respeito a questões de sustentabilidade global.

A fim de mitigar essa incerteza, os Estados utilizam da cooperação internacional para criar um caminho comum para lidar com problemas globais. Essa cooperação é uma das características da chamada Governança Global, fenômeno complexo que inclui atores públicos e privados, diversos interesses e influências, várias regras e estruturas de ação, e ideias conflitantes sobre o que a compõe ou não, tudo voltado para lidar com problemas comuns (ROEHRKASTEN, 2014). Ao longo dos anos, a Governança Global possibilitou o desenvolvimento ímpar do Direito Internacional (DI) e a emergência de vários regimes, especialmente aqueles de base ambiental, como é o caso do complexo de regimes da biodiversidade (ORSINI, 2017).

Esse complexo permitiu aos Estados estabelecerem agendas e regras para a questão da biodiversidade, embora nem sempre em harmonia. No entanto, houve uma grande lacuna neste complexo de regimes a respeito da biodiversidade marinha, notadamente aquela além da jurisdição nacional (BAJN), a qual ainda não é objeto do Direito Internacional de forma direta (ROCHETTE et al., 2015).

Com o objetivo de preencher essa lacuna, pactuou-se na cúpula da Rio+20, em 2012, pelo desenvolvimento de um documento internacional vinculante para abordar essa lacuna. Pode-se ligar essa decisão com o aumento da preocupação com questões oceânicas na governança ambiental global (MAZZEGA et al., 2018). Esta decisão é um marco para a governança global da biodiversidade. Sem dúvida, a questão da biodiversidade ${ }^{2}$ marinha está posta. Atualmente, encontra-se em negociação o acordo obrigatório sobre biodiversidade marinha, chamado BBNJ em inglês.

Pelo acima exposto, percebe-se também a necessidade da análise das dinâmicas globais que incidem sobre a questão da biodiversidade em alto-mar. Entende-se que a humanidade está em um momento crítico para a ação, tendo em vista que as consequências dessa (in)ação podem dar o tom da capacidade da atual e das futuras gerações em gozar do direito a uma vida digna, dado que o meio ambiente é responsável pela geração de serviços ecossistêmicos que sustentam a vida humana. Desta feita, a diplomacia brasileira poderia apresentar mecanismos efetivos de interação com esse sistema complexo de governança, de forma a atingir objetivos que possam tanto contribuir com a gestão sustentável da biodiversidade marinha global, tendo o DI um lugar de destaque, como favorecer a agenda social brasileira de desenvolvimento sustentável, principalmente para aqueles que necessitam desses recursos marinhos para a sua subsistência.

Neste contexto, a pesquisa tem como pergunta: como o Brasil poderia maximizar sua participação para promover seus interesses de país emergente e situado no Atlântico Sul?

Parte-se da premissa de que a governança global da biodiversidade em alto-mar é um sistema complexo que, consequentemente, precisa de uma resposta à altura. Esse artigo, portanto, faz parte

Maljean-Dubois (2016) aponta para o fato dessa governança ser não somente global, mas multiescalar, pois é transversal à todos os níveis, por isso também pertencendo à agenda global e internacional. 
de uma tentativa de integração do modelo de complexidade para o entendimento das dinâmicas globais sobre o meio ambiente, tendo como foco a atuação brasileira por meio de uma diplomacia estratégica para a questão da biodiversidade além das jurisdições nacionais.

O objetivo geral deste artigo, portanto, é analisar o processo da governança da biodiversidade em alto-mar. O objetivo específico é e o de analisar o que está em jogo para o Brasil.

A metodologia adotada é essencialmente qualitativa, baseando-se principalmente na técnica de pesquisa bibliográfica. As fontes utilizadas são principalmente running records, com eventual uso de episodic records (JOHNSON; REYNOLDS; MYCOFF, 2016). Mais especificamente, produção acadêmica e documentos oficiais desenvolvidos sobre a questão.

O texto está dividido em três partes: a primeira versa sobre a teoria da complexidade para as questões ambientais globais; a segunda sobre como utilizar esta perspectiva a partir do framework da diplomacia estratégica; por fim, aplica-se às duas primeiras seções para o caso das negociações em andamento do novo acordo para a biodiversidade em alto-mar (BAJN), focando na atuação brasileira.

\section{A governança global da biodiversidade em alto-mar}

Os oceanos são tanto capazes de separar como servir de ponte, por isso, estão presentes como questão entre unidades políticas há milênios ${ }^{3}$. São fontes de recursos, vivos (que estão a passar por um processo caracterizado de marine defaunation [MCCAULEY et al., 2015], ou seja, um processo de perda de biodiversidade) e não vivos; são meios de transporte, cerca de $80 \%$ do comércio mundial se dá por vias marítimas (UNITED..., 2015); são essenciais para a regulação climática global, porém não sem consequências, dadas as crescentes atividades humanas, o que resulta em aumento da acidificação dos oceanos, por exemplo (PERSHING et al., 2018).

Os oceanos fazem parte do que Kavalski (2016) chama de "vida global", sendo sua influência decisiva sobre a humanidade. De forma a dar sentido a esse espaço global salgado, que cobre cerca de $70 \%$ da superfície terrestre, o DI é uma das ferramentas utilizadas pelos para estabelecer princípios, normas, regras e procedimentos de tomada de decisão para reger a questão dos oceanos - ou seja, estabelecer regimes, a partir da definição clássica de Krasner (1982). ${ }^{4}$

Porém, não há um só regime sobre "a questão oceânica". O que existe é uma miríade de regimes inter-relacionados, cujo grau de densidade e efetividade de cada regime e de suas relações são variados, que atuam sobre temas sobrepostos, advindo de diversas fontes e, por vezes, apresentando estruturas autônomas. Alguns exemplos são: clima, pesca, transportes, energia, turismo e aquacultura.

Tal situação poderia ser compreendida, então, pela ideia de complexo de regimes, ou como Orsini et al. (2013, p. 29, traduzido pelo autor) definem:

uma rede de três ou mais regimes internacionais que se relacionam numa questão em comum, exibindo participação sobreposta e gerando interações substantivas,

\footnotetext{
3 Para uma discussão sobre a evolução da temática dos oceanos numa perspectiva histórica do direito internacional, ver Bederman (2012).

4 De acordo com o autor: "regimes can be defined as sets of implicit or explicit principles, norms, rules, and decision-making procedures around which actors' expectations converge in a given area of international relations" (KRANSER, 1982, p. 186).
} 
normativas, ou operativas reconhecidas como potencialmente problemáticas, quer sejam ou não gerenciadas de maneira efetiva. ${ }^{5}$

Um nódulo central para esse complexo de regimes é a Convenção das Nações Unidas sobre o Direito do Mar (CNUDM), de 1982, com entrada em vigor em 1994. Um dos objetivos dessa convenção é delinear o regime geral para os oceanos, de forma a harmonizar o DI para essa questão - em que pese esse objetivo ter sido alcançado à época de sua assinatura, há discordâncias (DUPUY, VIÑUALES, 2015; BEURIER, 2017). Um dos grandes desafios dessa convenção é a disposição sobre cerca de $60 \%$ da superfície terrestre não integrantes da soberania dos Estados: as zonas além das jurisdições nacionais (ZAJN).

Nas ZAJN dois são os espaços regulados: a Área, considerada patrimônio comum da humanidade $^{6}$, incluindo os fundos marinhos e seu subsolo, no qual os recursos minerais fazem parte do mesmo regime de gestão internacional; e as águas sobrejacentes, o alto-mar, onde os recursos biológicos são considerados res nullius e podem ser apropriados por qualquer Estado capaz de fazê-lo, apesar de nenhum Estado possuir direitos exclusivos sobre eles e terem o dever de cooperar (BARROS-PLATIAU; MALJEAN-DUBOIS, 2017).

Uma primeira dificuldade em utilizar a perspectiva dos regimes para os oceanos aparece nesse ponto, a do recorte do problema para a questão somente institucional. Como esse é um ambiente marinho complexo e de interação humana constante, o seu entendimento precisa partir de uma abordagem abrangente, capaz de integrar os diferentes níveis de interação em diferentes escalas, sejam eles naturais e/ou sociais. Além do mais, a perspectiva dos regimes não considera as interações além das suas bases de definição ${ }^{7}$, por esse motivo, esse ambiente marinho necessita ir além de um complexo de regimes e, nas palavras de Barros-Platiau e Maljean-Dubois (2017), precisa de uma abordagem de governança complexa.

A discussão na literatura de Relações Internacionais sobre governança global é extensa, e por questão de espaço, preferiu-se utilizar um artigo baseado numa revisão sistemática sobre a pesquisa na questão. Bennett e Satterfield (2018) desenvolvem um framework para a governança ambiental, baseada numa ampla revisão de literatura sobre o tema. De acordo com os autores, a governança ambiental pode ser caracterizada como "as instituições, estruturas e processos que determinam quem toma decisões, como e para quem as decisões são tomadas, se, como e quais ações são tomadas, e por quem e com que efeito", com o objetivo de "administrar comportamentos individuais ou ações coletivas em busca de bens ambientais públicos e resultados sociais relacionados" ${ }^{8}$ (BENNETT; SATTERFIELD, 2018, p. 2, 6, traduzido pelo autor).

\footnotetext{
Original: "a network of three or more international regimes that relate to a common subject matter; exhibit overlapping membership; and generate substantive, normative, or operative interactions recognized as potentially problematic whether or not they are managed effectively"

6 Conferir Oliveira e Maljean-Dubois (2015) para uma discussão sobre os conceitos de bem público mundial, patrimônio comum da humanidade e bens comuns sobre os recursos marinhos.

7 Mesmo que se reconheça a grande evolução nos estudos sobre regimes desde a definição clássica de Krasner (1982). Conferir Breitmeier et al. (2006), Inoue (2016), Young (2017) e Weiss (2013), por exemplo, para a expansão da noção de regimes, principalmente para o aspecto da sua expansão como conceito, mas continuada limitação para o foco nas relações entre os Estados. 8 Original: "the institutions, structures, and processes that determine who makes decisions, how and for whom decisions are made, whether, how and what actions are taken and by whom and to what effect", "manage individual behaviors or collective
} 
Assim, a governança global ambiental considera os processos formais e informais, bem como atores estatais e não-estatais, de diversas naturezas, que funcionam na base de uma autoridade diluída em diversas escalas (do local ao global, e também entre suas inter-relações), porém organizada, para atingir objetivos e reger determinadas questões-problema, como é o caso da biodiversidade em alto-mar. De certo modo, os regimes fazem parte da governança, porém esta última é mais ampla por ir além das relações interestatais e agregar outros atores, por exemplo. ${ }^{9}$

À primeira vista, na tentativa de leitura da governança global da biodiversidade em alto-mar, uma dualidade emerge: a da existência e inexistência de regulação a nível internacional. Essa dualidade é percebida pelos inúmeros acordos, organizações, programas e outros processos e estruturas que funcionam para regular esta questão, ou de maneira muito geral ou muito setorial, ao mesmo tempo inexiste um regime capaz de estabelecer definições e regras claras para as ações tomadas nessa área (BARROS-PLATIAU; MALJEAN-DUBOIS, 2017). Caracteriza-se, desse modo, uma fragmentação da governança para a biodiversidade em alto-mar.

A fragmentação é uma consequência da compartimentalização de determinados assuntos em categorias e setores, cujo objetivo é o de simplificação dos processos para melhor abordá-los. Esta também é uma característica da própria produção do Direito Internacional, onde cada tratado é uma realidade em si mesma, definindo suas regras para sua questão, e mesmo que haja ligações explícitas entres os diferentes regimes, cada qual possui partes distintas não necessariamente interligadas. Biermann et al. (2009) identificam três graus de fragmentação: 1) o da integração institucional e da sobreposição de sistemas de tomada de decisão; 2) a existência de conflito entre normas; 3) a constelação dos atores. Assim, a interação e a densidade desses graus são os indicadores do nível da fragmentação, seja do Direito Internacional ou da governança.

Esse fenômeno não deve ser visto como não intencional ou fruto do acaso, pelo contrário, resulta de interesses imediatos e individuais dos Estados, apesar de não serem os únicos atores na construção do DI e da governança (BARROS-PLATIAU; MALJEAN-DUBOIS, 2017). Apesar disso, a fragmentação não é inerentemente negativa. A sua existência é uma resposta à complexidade das relações internacionais, podendo variar de conflitiva à cooperativa, ou mesmo sinergética (BIERMANN et al., 2009).

No caso da governança global da biodiversidade em alto-mar está em curso uma tentativa de enfrentar o desafio da fragmentação dessa governança. Em 2004, a Assembleia Geral das Nações Unidas (AGNU) estabeleceu o Grupo de Trabalho BAJN, para o estudo de questões relacionadas à conservação e uso sustentável da biodiversidade marinha além da jurisdição nacional, de acordo com a Resolução 59/24 de 17 de novembro de 2014 da AGNU.

As discussões do grupo começaram em 2006 e terminaram em $2015^{10}$, neste período, as discussões focaram na fragilidade e nas lacunas do quadro regulatório internacional existente e se havia a necessidade da adoção de um novo instrumento sob a CNUDM para lidar com os problemas da biodiversidade além da jurisdição nacional. Deve-se ter em conta que a abertura das negociações

\footnotetext{
actions in pursuance of public environmental goods and related societal outcomes".

9 Ver Inoue (2016) para uma discussão da profunda relação entre regimes internacionais e governança global.

10 Para uma discussão sobre as pautas e decisões dessas reuniões, cf. Nunes (2017).
} 
é um marco, tendo em vista que os Estados estavam bastante divididos quanto ao próximo passo a se tomar: ou melhorar as já existentes instituições, ou adotar um novo instrumento (FREESTONE et al., 2014). Destacando-se o setor da pesca, em função da sua atual importância econômica e das obrigações internacionais já estabelecidas, por meio de OROPs (Organizações Regionais de Ordenamento Pesqueiro, RFMO em inglês) por exemplo.

Esse processo se consolidou principalmente depois de 2014, com a formação de uma coalizão a favor da abertura das negociações, composta por: Austrália, G77/China, México, Nova Zelândia e União Europeia, apoiados por grupos regionais como a Comunidade Caribenha, o Grupo de Pequenas Ilhas do Pacífico e a União Africana; enquanto o Canadá, os EUA e a Rússia mostraram-se relutantes em abrir as negociações (WRIGHT, et. al, 2014). Em 2015, recomendou-se para a AGNU o desenvolvimento de um instrumento internacional juridicamente vinculante sob a égide da CNUDM.

Já em 2015, a AGNU deu início ao processo de negociação desse novo tratado, estando atualmente em negociação, previstas para finalizarem em 2020 (BROGGIATO et al., 2017; INTERGOVERNMENTAL..., [s.d]). Os temas principais em negociação versam sobre: reforço de capacidades e transferência de tecnologia; instrumentos de gestão em área; estudos de impacto ambiental; e recursos marinhos genéticos, inclusive questões de repartição de benefícios (IISD, 2018).

Porém, diversas outras questões estão em aberto e podem ainda fazer parte das discussões, adicionando ainda mais níveis de complexidade. A questão da poluição marinha, principalmente de plásticos, tem sido uma questão altamente discutida e mesmo defendida como tema para o novo acordo, estando presente também no Objetivo de Desenvolvimento Sustentável 14 (ODS 14) "(AMARAL-ZETTLER, 2015; TYLLER, NYMAN, 2018). Já a discussão sobre bioprospecção e mineração, bem como da aplicação dos limites e definições de cada um desses regimes, está no centro da discussão sobre recursos genéticos, repartição de recursos e transferência de tecnologia, apresentando uma clara divisão Norte-Sul quanto à abordagem a ser adotada no novo acordo (BLASIAK et al., 2016; BROGGIATO et al., 2017; DRANKIER et al., 2012). Ricard (2017) e Druel et al. (2012) explicam que essa divisão já se cristalizou nas discussões durante o Grupo de Trabalho BAJN, baseado principalmente na questão econômica da biodiversidade: o Sul, concentrado em torno do Grupo dos 77 e China, defende que o princípio do patrimônio comum da humanidade também se estenderia aos recursos vivos da ZAJN, o que levaria a obrigatoriedade de partilha dos benefícios obtidos; enquanto o Norte, como os Estados Unidos, a Rússia, a Islândia, o Japão e mesmo a Noruega, defendem que não, os recursos vivos na ZAJN estariam sob a égide do princípio da liberdade nos mares.

Uma outra questão é a dos sistemas socioecológicos, nos quais a vulnerabilidade do sistema ecológico está diretamente ligada com aquela do sistema socioeconômico, tendo em vista a necessidade de pensar a interação entre os sistemas sociais e ecológicos na produção de políticas eficazes de conservação e preservação, bem como de adaptação das comunidades costeiras para os novos desafios postos graças às mudanças climáticas (METCALF et al., 2015). Assim, a abordagem ecos-

ODS 14, meta 14.1: "14.1 By 2025, prevent and significantly reduce marine pollution of all kinds, in particular from land-based activities, including marine debris and nutrient pollution", indicador 14.1.1: "Index of coastal eutrophication and floating plastic debris density" (SUSTAINABLE..., [s.d], online). 
sistêmica ${ }^{12}$ parece cada vez mais como uma necessidade (BARROS-PLATIAU; MALJEAN-DUBOIS, 2017) e de necessária inclusão no novo acordo.

As questões não se reduzem a estas, ou mesmo àquelas discutidas centralmente no processo de negociação; pode-se mesmo questionar sobre o papel da ciência ou da privatização neste processo (HARDEN-DAVIES et al., 2017; BLASIAK et al., 2018). Portanto, a questão da BAJN é complexa, havendo a necessidade de uma governança capaz de lidar com seus desafios.

Abaixo explica-se o que se quer dizer com o entendimento de uma questão a partir de uma lente da "complexidade".

\section{Uma abordagem complexa para um ecossistema complexo}

A complexidade tem sido empregada cada vez mais no vocabulário das Relações Internacionais como um conceito operacional. Entretanto, para compreender o seu emprego, vale retomar como a literatura aplica essa teoria para a governança global. Nesta seção, propõe-se uma abordagem centrada na complexidade como framework (quadro de análise) e na diplomacia estratégica como modo de ação.

Adotar uma postura de leitura por meio da complexidade é adotar uma abordagem sistêmica, onde a análise de partes de um sistema dá lugar para a análise das interações entre diferentes níveis (local, nacional, internacional...) e fontes (biológicas, econômicas, sociais...). Assim, entende-se que há uma irredutibilidade dessas interações, por uma oposição quanto a tentativas de simplificação dos processos, já que essa cadeia de relações é caracterizada principalmente pela incerteza, seja das suas causas, resultados, fluxos ou impactos (LE PRESTRE, 2017). Este último argumento é ilustrado pela diferença entre problemas "complexos" e "complicados". Como Ho (2017) explica, sistemas "complicados" possuem características newtonianas, já que executam funções pré-determinadas que são predizíveis e repetíveis, onde cada nova entrada (input) leva a um resultado esperado (output). Destarte, algo complicado pode ser dividido em partes menores e mais fáceis de manipular (LE PRESTRE, 2017). Já sistemas "complexos" apresentam características contrárias, onde a repetibilidade e a predeterminação não têm lugar, pois são sistemas com diversas partes autônomas, porém conectadas de diversas formas, fazendo com que sua redução seja na verdade uma distorção, já que o comportamento do sistema não pode ser estudado por meio das suas partes isoladas (HO, 2017; LE PRESTRE, 2017).

A Tabela 1 abaixo resume as características e propriedades de um sistema complexo.

12 De acordo com o Report of the Workshop on the Ecosystem Approach: "The ecosystem approach is based on the application of appropriate scientific methodologies focused on levels of biological organization which encompass the essential processes and interactions amongst organisms and their environment. The ecosystem approach recognizes that humans are an integral component of ecosystems" (FINAL..., 1998, p. 3). 
Tabela 1. Características e propriedades de um sistema complexo

\begin{tabular}{|c|c|}
\hline \multicolumn{2}{|c|}{ Sistema Complexo } \\
\hline Características & Propriedades \\
\hline $\begin{array}{l}\text { Múltiplos elementos (agentes ou unidades) de várias } \\
\text { espécies; }\end{array}$ & Auto-organização e autorregulação; \\
\hline $\begin{array}{l}\text { Intrinsecamente interconectados uns aos outros, } \\
\text { formando redes, o que leva às características abaixo; }\end{array}$ & Emergência; \\
\hline $\begin{array}{l}\text { Não-linearidade e circuitos de retorno (feedback } \\
\text { loops); }\end{array}$ & $\begin{array}{l}\text { Dinâmica multinível, decorrente da hierarquia, o que } \\
\text { requer uma governança interescalar; }\end{array}$ \\
\hline Hierarquia, o mundo é multiescalar; & Consequências não involuntárias; \\
\hline \multirow{5}{*}{$\begin{array}{l}\text { Abertura, há uma troca de energia e informação } \\
\text { com o seu ambiente. }\end{array}$} & $\begin{array}{l}\text { Irreversibilidade (pontos de virada (tipping points) } \\
\text { para novos regimes) e não renovabilidade (como } \\
\text { esgotamento de recursos); }\end{array}$ \\
\hline & Adaptabilidade; \\
\hline & Surpresas e catástrofes, múltiplos futuros; \\
\hline & $\begin{array}{l}\text { Dependência de trajetória (path dependency), história; o } \\
\text { comportamento de um sistema complexo é contingente } \\
\text { no contexto local e na sequência de eventos; }\end{array}$ \\
\hline & Robustez, resiliência e sustentabilidade. \\
\hline
\end{tabular}

Fonte: produzido pelo autor, baseado em Le Prestre (2017, p. 135).

Vale esclarecer alguns pontos levantados pela Tabela 1, nem todos serão abordados já que suas explicações estão contidas na tabela acima. A multiplicidade de elementos é tanto caracterizada pela quantidade, como também pela natureza do processo, destacando a privatização, caracterizado pela emergência de atores não-estatais, tais como ONGs e empresas, onde há certo nível de autoridade ${ }^{13}$ (GREEN; AULD, 2016), e pela diversidade deles, em crescente interação.

$O$ fato de formarem redes faz com que os sistemas complexos apresentem as características da coluna à esquerda. A não-linearidade é explicada por Kavalski (2016, p. 64, traduzido pelo autor) como aquela "cuja dinâmica não é expressável como a soma do comportamento de suas partes"14. Nas palavras de Le Prestre (2017), há uma desproporcionalidade entre causa e efeito, entradas e saídas. Como parte dessa característica, qualquer mudança no sistema pode levar a grandes mudanças, o que é leva às ideias de "cisnes negros" (black swans), efeito borboleta e elefante negro

13 De acordo com Green e Auld (2016), autoridade privada ocorre em situações em que atores não-estatais definem um conjunto de regras ou padrões em que outros atores na política internacional adotam.

14 Original: "whose dynamics are not expressible as a sum of the behaviors of its parts". 
(black elephant) ${ }^{15}(\mathrm{HO}, 2016)$. Já os feedbacks são a característica de reflexividade do sistema, esses retornos podem ser positivos (tendendo a desestabilização, caos) ou negativos (tendendo a estabilização, ordem) (LE PRESTRE, 2017; KAVALSKI, 2016). A hierarquia pressupõe a existência de entidades em níveis organizacionais inter-relacionados, onde os sistemas podem cruzar diversos níveis, criando um fluxo simultâneo top-down e bottom-up (LE PRESTRE, 2017).

Apesar de serem complexos, esses sistemas não são desordenados ou caóticos, pois apresentam a capacidade de auto-organização, definida como "a criação espontânea de um padrão coerente a partir de interações locais' ${ }^{16 ”}$, um processo naturalmente bottom-up (KAVALSKI, 2016, p. 64, traduzido pelo autor). Já a emergência somente é perceptível tendo o sistema como um todo. Le Prestre (2017, p. 142, traduzido pelo autor) explica que essa propriedade "refere-se ao surgimento de estruturas, padrões, propriedades ou processos inesperados e complexos a partir de interações simples em um sistema auto-organizado"7". Parafraseando Ho (2014, p. 4), quando algo "emerge", a surpresa é presente.

Por fim, vale recorrer a Young (2010) para a definição de robustez e resiliência. O autor considera robustez como "a capacidade do sistema de lidar eficazmente com os desafios e tensões sem sofrer mudanças significativas em seus próprios elementos ou processos", e resiliência como "a capacidade de um sistema de experimentar distúrbios e ainda manter contínuos suas funções e controles contínuos" ${ }^{18}$ (YOUNG, 2010, p. 4-5, traduzido pelo autor). Já a estabilidade é caracterizada pela manutenção das estruturas regedoras do sistema no caso de perturbações, sejam internas ou externas.

Bai et al. (2016), por exemplo, aplicam esse framework para uma proposta de agenda de pesquisa voltada para a interação com os futuros possíveis do Antropoceno, com o objetivo atingir "o futuro que queremos", ilustrando a aplicação da teoria.

15 Cisnes negros: "one class of such surprises — rare and hard-to-predict events... their impact is large and game-changing"; efeito borboleta: "is the concept that small changes in initial conditions can produce large effects in a complex system"; elefantes negros: "the black elephant is a problem that is actually visible to everyone, but no one wants to deal with it, and so they pretend it is not there. When it blows up as a problem, we all feign surprise and shock, behaving as if it were a black swan" (HO, 2017, p. 4-6, 12).

16 Original: "the spontaneous creation of a coherent pattern out of local interactions".

17 Original: "refers to the rise of unexpected and complex structures, patterns, properties, or processes from simple interactions in a self-organizing system".

18 Original: "the capacity of a system to cope effectively with challenges and stresses without undergoing significant changes in its own elements or procedures"; "capacity of a system to experience disturbance and still maintain its ongoing functions and controls". 
Figura 1. Exemplo "Societal goals: the future we want"

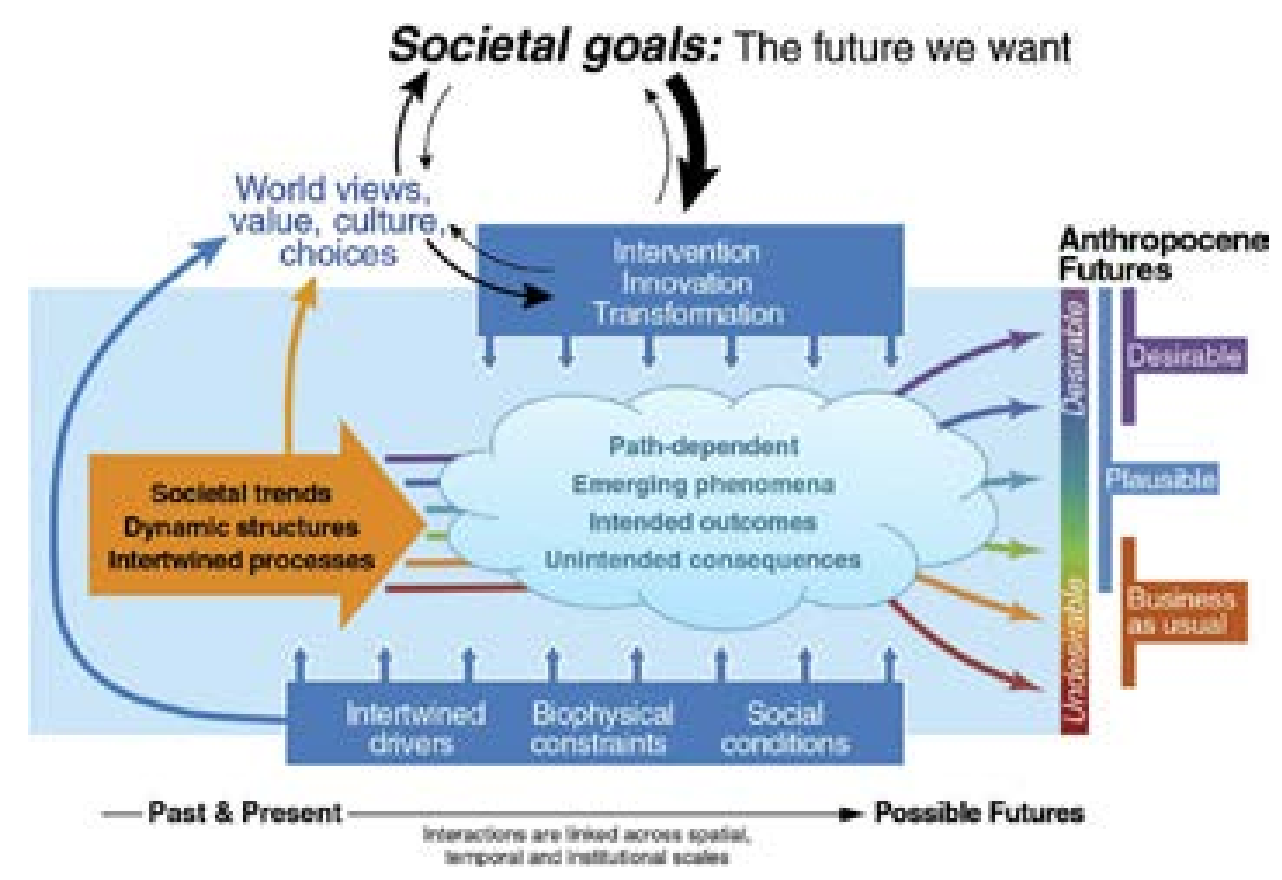

Fonte: Bai et al. (2016, p. 353).

Partindo da abordagem da complexidade, Prantl e Goh (2016) e Goh e Prantl (2017) desenvolvem a ideia de diplomacia estratégica. Smith (2016) também desenvolve uma abordagem com o mesmo nome, porém suas bases diferem dos autores anteriores.

Smith (2016) caracteriza uma diplomacia estratégica como um padrão de diplomacia centrada na busca de uma visão estratégica e apta a propiciar princípios e diretrizes para moldar o posicionamento do Estado tanto no nível global, quanto em relação aos seus principais parceiros estratégicos. Para tanto o autor enumera algumas condições para uma diplomacia estratégica: a) uma base institucional estável; b) meios de extrair e coordenar recursos relevantes; c) uma estratégia ou narrativa unificada estratégica; d) a capacidade de adaptar essa estratégia ou narrativa face às tendências internacionais; e) a capacidade de priorizar e almejar as ações de maneira coordenada sobre as principais relações, parceiros e atividades (SMITH, 2016). Assim, o autor conclui que caso essas características estejam presentes, os componentes principais de deliberação, representação, comunicação e negociação podem ser reunidos para formar uma diplomacia estratégica (SMITH, 2016).

A abordagem de Prantl e Goh (2016) e Goh e Prantl (2017) já é bem mais próxima da teoria da complexidade. Para os autores, a diplomacia estratégica é uma ferramenta para navegar no sistema, não focado em relações diádicas ou poliádicas, servindo tanto como uma ferramenta de diagnóstico, como de política públicaำ. Os autores complementam:

Estratégia implica vincular formas e meios para alcançar objetivos específicos, enquanto diplomacia é um meio vital pelo qual um Estado navega em seus caminhos escolhidos em direção aos fins das suas políticas públicas identificadas. A diplomacia

19 Optou-se traduzir policy para política pública. Para uma discussão sobre política externa e política pública cf. Milani e Pinheiro (2013). 
estratégica é definida como o processo pelo qual os atores estatais e não-estatais constroem e enquadram socialmente sua visão de mundo; definem suas agendas; e comunicam, contestam e negociam interesses e objetivos centrais divergentes (PRANTL; GOH, 2016, on-line, traduzido pelo autor) ${ }^{20}$

Logo, a construção da diplomacia pressupõe objetivos, construídos coletivamente, mas capazes de serem reacomodados com mudanças advindas do sistema. Deve-se notar os elementos da diplomacia estratégica desses autores: a) é uma diplomacia de longo termo com implicações sistêmicas; b) com um raciocínio estratégico forte com o objetivo de manutenção ou mudança do sistema; c) a curto prazo, contesta e negocia ideias e prioridades estratégicas conflitantes. O processo de construção de diplomacia estratégica pretende responder pelo menos três perguntas: 1) quais são os pontos de entrada (entry points) para a diplomacia estratégica?; 2) quais são os pontos de virada (tipping points) ${ }^{21}$, se existirem, que podem ou manter ou mudar o sistema?; 3) quais são os objetivos (end points) da diplomacia estratégica ao abordar as questões? (PRANTL; GOH, 2016).

Fica claro que a diplomacia estratégica deve estar enquadrada num âmbito muito maior, no qual a própria produção da política externa como um todo deve ser enquadrada. No entanto, seguindo os autores, existe a possibilidade de produção de diplomacias estratégicas setoriais $(\mathrm{GOH}$; PRANTL, 2017). Por esse motivo, o presente trabalho foca na possível construção de uma diplomacia estratégica oceânica brasileira voltada para os recursos marinhos biológicos além da jurisdição nacional. Porém, não se descarta a ideia de que essa diplomacia setorial deveria estar alinhada com os objetivos gerais de uma diplomacia estratégica geral.

Para esclarecer a ligação entre a necessidade de uma diplomacia estratégica para atuar em um sistema complexo, como é o caso da governança global da biodiversidade em alto mar, recorre-se a Le Prestre (2017, p. 137, traduzido pelo autor):

Em um sistema complexo, onde a incerteza sobre o comportamento de outros agentes, a natureza do problema e as conseqüências de suas ações prevalecem, os agentes adotam estratégias criadas para garantir o acesso aos recursos necessários, maximizar a flexibilidade futura e minimizar as perdas imediatas. Eles podem buscar um bem comum bem definido (ou seja, uma noção clara de equilíbrio desejável), mas cada classe de agente irá concebê-lo e abordá-lo de maneira diferente ${ }^{22}$.

Assim, adotar uma postura frente a um sistema complexo é se preparar frente aos seus desafios e mudanças recorrentes. Portanto, a não adoção de uma ação estratégica é se dispor a ser somente

20 Original: "Strategy entails linking ways and means to achieve specific goals, while diplomacy is a vital means by which a state navigates its chosen paths toward its identified policy ends. Strategic diplomacy is defined as the process by which state and non-state actors socially construct and frame their view of the world; set their agendas; and communicate, contest and negotiate diverging core interests and goals".

21 Barros-Platiau et al. (2018, p. 71) caracterizam pontos de virada como: "tipping points can be described as the instance at which an event becomes the catalyst of broader causal dynamics throughout the system, the conjunctional impact of which works to bring about the intended end point".

22 Original: "In a complex system, where uncertainty regarding the behavior of other agents, the nature of the problem, and the consequences of one's actions prevail, agents adopt strategies designed to ensure access to needed resources, maximize future flexibility, and minimize immediate losses. They may pursue a well-defined common good (i.e. a clear notion of desirable equilibria), but each class of agent will conceive and approach it differently." 
reativo frente aos fluxos do sistema, de forma que as respostas não estariam baseadas em premissas ou objetivos claros, o que pode levar a um total fracasso da resposta. Algo muito arriscado no Antropoceno, tanto para o meio ambiente como para as pessoas.

A ideia da diplomacia estratégica não é criar algo absolutamente novo, muito pelo contrário, é utilizar dos recursos disponíveis ${ }^{23}$, que também já formam um sistema, mas definindo uma ação na tentativa de minimizar o espectro de possíveis feedbacks de forma a produzir um a política externa robusta, resiliente e estável.

Esclarece-se que a diplomacia estratégica não visa à mudança das práticas diplomáticas per se; a adaptação não é do comportamento, mas das estratégias (LE PRESTRE, 2017). Deve-se notar que a adoção de uma diplomacia estratégica não significaria uma tendência geral a essa mudança, isto é, a adoção de uma postura semelhante por parte dos outros atores. Porém, é somente quando essa adaptação ocorre entre os elementos do sistema que ele pode se tornar adaptativo para a mudança de posicionamento (LE PRESTRE, 2017).

Concluindo esta seção, pode-se dizer que o uso do framework aqui apresentado é benéfico no sentido em que ele oferece ferramentas, tanto analíticas, como de formulação de políticas, para abordar a dinamicidade e imprevisibilidade da vida global (KAVALSKI, 2016). Logo, a complexidade inerente à questão da governança global da biodiversidade em alto-mar demanda uma resposta estruturada, e a diplomacia estratégica apresenta-se como uma possível resposta ao entendimento da política externa brasileira quanto a essa questão, principalmente em frente aos desafios postos pelo Antropoceno.

\section{As bases da ação: a diplomacia estratégica}

Uma pergunta natural decorrente é: por que o Brasil deve investir seus recursos na questão da biodiversidade além da jurisdição nacional? A principal é a falta de uma ação estratégica brasileira voltada para a BAJN (ver abaixo). Tendo em vista as oportunidades oferecidas pelos oceanos tanto para o desenvolvimento sustentável, quanto oportunidades científicas/tecnológicas e de inovação (OECD, 2019), o Brasil pode contribuir e sustentar a construção dessa agenda ao participar dela ativamente em busca de seu desenvolvimento sustentável.

Longe de ser um problema puramente ambiental, a BAJN é um problema multifacetado, sendo não só uma questão de regulação internacional, afetando modos de vida em vários níveis. Assim como durante a Rio-92, em que "os diplomatas brasileiros sabiam que a participação ativa era a melhor estratégia para proteger seus interesses nacionais e soberania" (BARROS-PLATIAU, 2010, p. 76, traduzido pelo autor), ${ }^{24}$ pode-se entender que a melhor estratégia é a participação, e se possível, com respaldo científico reconhecido mundialmente.

Abaixo, na Tabela 2, apresenta-se uma síntese da diplomacia estratégica.

23 O uso desses recursos leva a uma trajetória de dependência (path dependency), ou seja, as respostas dadas pela diplomacia estratégica refletem as características e o contexto no qual ela foi formulada. Daí a necessidade de uma construção coletiva. Ver próxima seção.

24 Original: "Brazilian diplomats knew that active participation was the best strategy to protect its national interests and sovereignty". 
Tabela 2. Síntese da diplomacia estratégica.

\begin{tabular}{|c|c|c|c|c|c|}
\hline \multicolumn{6}{|c|}{ Diplomacia Estratégica } \\
\hline Definiç̧̃å & \multicolumn{5}{|c|}{$\begin{array}{l}\text { É um padrão de diplomacia baseada numa visão estratégica, conectando caminhos e meios } \\
\text { para alcançar objetivos específicos, construída a partir de um processo em que atores estatais } \\
\text { e não-estatais constroem e enquadram sua visão de mundo, definem sua agenda, comunicam, } \\
\text { contestam e negociam diferentes interesses e objetivos, propiciando princípios e diretrizes } \\
\text { para moldar o posicionamento do Estado, tanto a nível global como em relação aos seus } \\
\text { parceiros e interesses estratégicos. }\end{array}$} \\
\hline Elementos & $\begin{array}{l}\text { Institucionalidade } \\
\text { estável }\end{array}$ & $\begin{array}{l}\text { Gestão de } \\
\text { recursos efetiva }\end{array}$ & $\begin{array}{l}\text { Narrativa } \\
\text { unificada }\end{array}$ & Adaptabilidade & Ação coordenada \\
\hline Condições & $\begin{array}{l}\text { Deliberação e } \\
\text { comunicação }\end{array}$ & $\begin{array}{l}\text { Representação e } \\
\text { negociação }\end{array}$ & $\begin{array}{l}\text { Foco a } \\
\text { longo termo } \\
\text { e com } \\
\text { implicações } \\
\text { sistêmicas }\end{array}$ & $\begin{array}{l}\text { Foco no curto } \\
\text { prazo deve ser } \\
\text { a construção } \\
\text { das prioridades }\end{array}$ & $\begin{array}{l}\text { Foco na } \\
\text { manutenção } \\
\text { ou mudança do } \\
\text { sistema }\end{array}$ \\
\hline Construção & $\begin{array}{l}\text { Quais são os } \\
\text { pontos de entrada? }\end{array}$ & $\begin{array}{l}\text { Quais são os } \\
\text { pontos de } \\
\text { virada? }\end{array}$ & $\begin{array}{l}\text { Quais são os } \\
\text { objetivos? }\end{array}$ & & \\
\hline
\end{tabular}

Fonte: produzido pelo autor, com base em Prantl e Goh (2016), Goh e Prantl (2017) e Smith (2016).

Com base na leitura da tabela, percebe-se que o objetivo de uma diplomacia estratégica é definir a problemática a partir das realidades de determinado Estado, no caso o Brasil, no sentido de não somente ser reativo às iniciativas de outros atores, mas também de oferecer soluções que são fruto de uma experiência única. Assim, adotar essa postura é uma resposta direta ao que Le Prestre (2017, p. 6, traduzido pelo autor) explica sobre os países em desenvolvimento nas questões ambientais: "eles falharam largamente em desenvolver definições do problema e oferecer soluções que não são reativas às iniciativas dos países desenvolvidos"25.

Ademais, a natureza da BAJN faz com que os atores capazes de ali atuarem tenham mais visibilidade, levando em conta a dificuldade tecnológica de realizar procedimentos de exploração, por exemplo. Porém, tendo em vista que a política externa é uma política pública, deve-se levar em conta que a mobilização de recursos do Estado deve visar tanto sua projeção internacional, bem como seu desenvolvimento, principalmente no caso brasileiro.

Pode-se perguntar: Brasil apresenta uma diplomacia estratégica para a BAJN? Se não, como construí-la? Em relação aos elementos dessa diplomacia, pode-se dizer que a PEB possui capacidade para ser institucionalmente estável, logo, também para apresentar uma visão a longo prazo, porém os outros elementos poderiam ser melhor trabalhados.

25 Original: "they have largely failed to develop definitions of the problem and offer solutions that are not reactive to developed countries' initiatives". 
A nível federal, a CIRM (Comissão Interministerial para os Recursos do Mar) é a instância que, nas atuais estruturas, melhor poderia servir aos interesses de uma diplomacia estratégica, principalmente por objetivar uma ação coordenada entre diversos ministérios. A utilização desse recurso pode ser contestada por essa estrutura levar em conta interesses além da política externa. Todavia, como Ho (2017) elucida, não é possível separar a condução desta política de outros interesses nacionais, por isso a necessidade de coordenação interna e troca de informações, o que torna a CIRM um possível fórum de discussão.

Contudo, para a existência de uma gestão de recursos efetiva e de uma narrativa unificada, essa Comissão precisa se adaptar para ser capaz de abrir para deliberação e comunicação, apresentando representação de diversos setores da sociedade (apesar de a comissão eventualmente receber contribuições externas), aptos a negociar suas visões conflitantes a curto prazo para a construção de uma estratégia a longo prazo, seja com o foco na mudança ou manutenção do sistema.

Essa deliberação e negociação são o que vão alimentar a produção da diplomacia estratégica de informações para serem sustentadas durante as negociações. A gestão de redes baseada no processo de mobilizar, traduzir, negociar, sintetizar e aplicar sistemas de conhecimento para a produção de políticas, pode servir para dinamizar as entradas da produção da diplomacia estratégica e atingir resultados bem-sucedidos (KERVRAN, PEPIN-LEHALLEUR, 2012; TENGÖ et al., 2017).

Quais seriam os pontos de entrada para o Brasil? Claramente, as negociações para o novo acordo da BAJN constituem o ponto principal para essa diplomacia focada na biodiversidade em alto-mar. A atuação do país depende do entendimento da sua posição no mundo, principalmente como "país ponte" entre o Norte e o Sul (BURGES, 2013), bem como da sua posição geográfica e de seus interesses nacionais. Desta forma, a cooperação com países do Atlântico parece ser essencial26, porém não se limitando a essa esfera geográfica, já que mesmo os BRICS podem se apresentar como de possível mobilização nas negociações, tendo em vista sua composição.

Ademais, a cooperação científica internacional, como a desenhada pela Declaração de Belém²7, é um importante subsídio para a posição do Brasil para essas negociações, contribuindo tanto para a construção da diplomacia, quanto canal de entrada e de diálogo entre os países para melhor compreender as necessidades dos oceanos e as oportunidades por eles oferecidas. Young et al. (2014, p. 396, traduzido pelo autor) elucidam a importância da ligação entre a ciência e o processo de formação de políticas:

Apoiar explicitamente o pensamento estratégico conjunto - incluindo o trabalho de longo prazo para a sustentabilidade - pode ajudar a identificar oportunidades para conectar agendas políticas e científicas, levar a uma melhor compreensão do

\footnotetext{
26 Por exemplo, conferir a Declaração de Brasília da IX Conferência de Chefes de Estado e de Governo da Comunidade dos países de língua portuguesa: "Reiteraram a importância do tema dos oceanos para os Estados-Membros da CPLP e, nesse sentido, reafirmaram o compromisso com a negociação de novo acordo internacional juridicamente vinculante, no âmbito da Convenção das Nações Unidas sobre o Direito do Mar (CNUDM), sobre a conservação e uso sustentável da biodiversidade marinha além das jurisdições nacionais" (MINISTÉRIO..., 2016, online).

27 A Declaração de Belém sobre a cooperação em investigação e inovação no oceano Atlântico é um documento assinado entre o Brasil conjuntamente com a União Europeia e a África do Sul, reconhecendo tanto o papel do Brasil bem como da ciência para os assuntos relacionados aos oceanos e também à Antártica, destacando-se o enfoque sobre reforço de capacidades e transferência de tecnologia, ponto principal da divisão Norte-Sul nas negociações do novo acordo, como já explicado acima (DECLARAÇÃO..., 2017). Essa Declaração é um claro exemplo de cooperação científica internacional (HARDEN-DAVIES, 2017).
} 
que a ciência pode oferecer dentro de um determinado período e reduzir os riscos de negligenciar questões emergentes ${ }^{28}$.

Como pontos de virada, aponta-se duas questões-chave: a emergência de novos temas (com o foco na poluição marinha, principalmente por plásticos) e a entrada de novos atores (com o foco na construção de capacidades e transferência de tecnologias [CC\&TT], notadamente o acesso a recursos genéticos marinhos).

A questão da poluição marinha por plásticos é uma agenda global, em que diversos países têm participação ativa, principalmente por meio de banimento de uso desses materiais ou na busca de desenvolvimento de novas tecnologias para substituir o seu uso, como no caso da União Europeia ou mesmo em alguns estados brasileiros (CAMARGO, 2018; EU..., 2018; LUIZ, CASSELA, 2019). A entrada desse tema nas negociações pode ser um catalisador para a mudança na abordagem para a biodiversidade em alto-mar, tendo em vista que isto poderia fortalecer a perspectiva ecossistêmica para a BAJN e, por consequência, também fortalecer a ligação entre esse regime e outros sobre a biodiversidade, contribuindo para a sua desfragmentação. Também, fortaleceria uma ação entre as redes de cientistas para encontrar soluções para a poluição por plástico, abrindo portas para novas tecnologias de produção e de manejo desses materiais - ou seja, ciência/tecnologia e inovação (OECD, 2019).

Logo, o segundo ponto de virada está inerentemente ligado ao primeiro. Contudo, a questão apresenta uma dificuldade vinculada ao conflito Norte-Sul. Isto se dá pela preponderância de pouco mais de 10 países desenvolvidos na liderança das pesquisas sobre biodiversidade em alto-mar, principalmente a relacionada sobre recursos genéticos. A empresa alemã BASF, por exemplo, registrou cerca de $47 \%$ de todas as patentes sob proteção internacional associadas a recursos marinhos genéticos entre o período de 1988 e 2017 (BLASIAK et al., 2018). Assim, a CC\&TT não pode ser pensada somente a nível estatal ou de cooperação científica internacional, precisaria incluir atores privados, que controlam grande parte das operações em alto-mar sobre a biodiversidade. Este é um desafio mesmo para o Direito Internacional, tendo em vista a relutância dos Estados de reconhecer esses atores como sujeitos do DI. O resultado dessa discussão pode certamente mudar os rumos das negociações.

Se no caso das negociações das mudanças climáticas o objetivo é "salvar o clima" (BARROSPLATIAU et al., 2018), para a ZAJN é "salvar os oceanos". Como na quarta revolução industrial, onde o conhecimento tem papel fundamental, o desenvolvimento da ciência é justamente aquilo que capacita a atuação em campos complexos, principalmente para a ZAJN. Fica claro que os oceanos são oportunidade de desenvolvimento para a ciência, e também para a população brasileira, concentrada principalmente nas costas oceânicas. Portanto, "salvar os oceanos" é oferecer um "espaço seguro para a humanidade" dentro das fronteiras planetárias (BARROS-PLATIAU et al., 2018; BIERMANN, 2012; ROCKSTRÖM et al., 2009).

A tabela 3 abaixo traz o resumo da discussão acima, apontando para o fato que diplomacia brasileira ainda fica para trás no quesito estratégico para a biodiversidade além da jurisdição nacional.

28 Original: "Supporting joint strategic thinking explicitly-including work on long term visions for sustainability-can help to identify opportunities to connect science and policy agendas, lead to a better understanding of what science might be able to offer within a particular timeframe, and reduce the risks of neglecting emerging issues." 
Tabela 3. Resumo da Diplomacia Estratégica brasileira para a BAJN

\begin{tabular}{|c|c|c|c|c|c|}
\hline \multicolumn{6}{|c|}{ Diplomacia estratégica brasileira para a BAJN } \\
\hline Elementos & $\begin{array}{l}\text { Possui uma } \\
\text { institucionalidade } \\
\text { estável }\end{array}$ & $\begin{array}{l}\text { Gestão de } \\
\text { recursos } \\
\text { ainda não é } \\
\text { totalmente } \\
\text { efetiva }\end{array}$ & $\begin{array}{l}\text { Não apresenta } \\
\text { políticas para a } \\
\text { BAJN, logo não } \\
\text { apresenta uma } \\
\text { narrativa unificada }\end{array}$ & $\begin{array}{l}\text { Adaptabilidade } \\
\text { presente, } \\
\text { podendo integrar } \\
\text { outros atores }\end{array}$ & $\begin{array}{l}\text { Ação } \\
\text { coordenada } \\
\text { baseada na } \\
\text { CIRM }\end{array}$ \\
\hline Condições & $\begin{array}{l}\text { Deliberação e } \\
\text { comunicação } \\
\text { precisam ser } \\
\text { reforçadas }\end{array}$ & $\begin{array}{l}\text { Representação } \\
\text { existe para } \\
\text { alguns setores } \\
\text { e negociação } \\
\text { precisa ser } \\
\text { reforçada }\end{array}$ & $\begin{array}{l}\text { Para haver um } \\
\text { foco a longo } \\
\text { termo e com } \\
\text { implicações, } \\
\text { deve-se desenhar } \\
\text { uma política clara, } \\
\text { ainda inexistente }\end{array}$ & $\begin{array}{l}\text { Foco no curto } \\
\text { prazo não está } \\
\text { na construção } \\
\text { das prioridades, } \\
\text { mas sim nas } \\
\text { negociações do } \\
\text { novo acordo }\end{array}$ & $\begin{array}{l}\text { Foco na } \\
\text { manutenção, } \\
\text { dada a tradição } \\
\text { diplomática } \\
\text { de apoio ao } \\
\text { multilateralismo }\end{array}$ \\
\hline Construção & $\begin{array}{l}\text { Pontos de } \\
\text { entrada: a } \\
\text { negociação do } \\
\text { novo acordo } \\
\text { para a BAJN; } \\
\text { cooperação } \\
\text { internacional } \\
\text { para questões } \\
\text { oceânicas; e } \\
\text { cooperação } \\
\text { científica } \\
\text { internacional }\end{array}$ & $\begin{array}{l}\text { Pontos } \\
\text { de virada: } \\
\text { privatização } \\
\text { e poluição } \\
\text { marinha } \\
\text { (plásticos) }\end{array}$ & $\begin{array}{l}\text { Objetivos: } \\
\text { proporcionar o } \\
\text { desenvolvimento } \\
\text { sustentável } \\
\text { a partir da } \\
\text { conservação e uso } \\
\text { sustentável dos } \\
\text { recursos vivos na } \\
\text { ZAJN }\end{array}$ & & \\
\hline
\end{tabular}

Fonte: produzido pelo autor.

\section{Considerações finais}

Este artigo é uma tentativa de diálogo entre teoria e prática para a questão da biodiversidade em alto-mar. Como argumento, expõe-se que a questão é altamente complexa e, por esse motivo, a teoria da complexidade oferece um grau analítico elevado para o entendimento da questão. Todavia, este entendimento não deve se passar somente pela questão analítica, tendo em vista a necessidade de ação sobre esse ecossistema frágil e ainda com muito a ser descoberto. Assim, oferece-se a abordagem da diplomacia estratégica, arraigada na teoria da complexidade, para formular uma resposta estruturada e capaz de utilizar de forma racional e estratégica recursos escassos para atingir necessidades de desenvolvimento sustentável.

Adotar uma postura de diplomacia estratégica é olhar para o "internacional" como um sistema complexo, perguntando-se onde o país "encaixa-se" nesse sistema, como agir, quais são seus objetivos, e como alcançá-los. De fato, é como estar imerso no oceano: um ambiente em que cada 
movimento tem uma reverberação, ao qual outros percebem e reagem ou são proativos; não se sabe muito bem os resultados, por isso, estar preparado para as diversas consequências é essencial. No caso brasileiro, caberia o uso de uma diplomacia estratégica para a defesa das prioridades dos países em desenvolvimento.

A tradução dessa proposta para a realidade é desafiadora e deixa certas questões em aberto: qual a diferença para uma diplomacia tradicional (GOH; PRANTL, 2017)? Seria de fato uma alternativa custo efetiva? O modus operandi da diplomacia, apesar da sua institucionalidade, também é afetada pelas linhas da política externa adotada pelo governo, como alinhar à perspectiva de longo prazo da diplomacia estratégica? Quais as consequências de adotar uma postura estratégica enquanto os outros adotam uma tradicional? Essas e outras questões devem ser fonte de discussões futuras.

\section{Referências}

ANGELO, Claudio. Acordo de Paris começa, enfim, a ganhar cara. Observatório do clima, 05 out. 2015. Disponível em: http://www.observatoriodoclima.eco.br/acordo-de-paris-comeca-enfim-a-ganhar-cara/. Acesso em: 11 fev. 2019.

AMARAL-ZETTLER, Linda A. et al. The biogeography of the Plastisphere: implications for policy. Frontiers in Ecology and the Environment, [S. l.], v. 13, n. 10, p. 541-546, 2015. https://doi. org/10.1890/150017

BAl, Xuemei et al. Plausible and desirable futures in the Anthropocene: A new research agenda. Global Environmental Change, Guildford, v. 39, p. 351-362, 2016. https://doi.org/10.2989/16073 $\underline{606.2015 .1073813}$

BARROS-PLATIAU, A. F. et al. Correndo para o mar no antropoceno: a complexidade da governança dos oceanos e a estratégia brasileira de gestão dos recursos marinhos. Revista de Direito Internacional, Brasília, DF, v. 12, n. 1, p. 150-168, 2015 https://doi.org/10.5102/rdi.v12i1.3292

BARROS-PLATIAU, Ana Flávia; MALJEAN-DUBOIS, Sandrine. La gouvernance globale de la biodiversité en haute mer. In: COMPAGNON, Daniel; RODARY, Estienne. Les Politiques de biodiversité. Paris: Presses de Sciences Po, 2017. p. 49-66.

BARROS-PLATIAU, Ana Flávia et al. Strategic Diplomacy, Fractal Governance and the BRICS in the Climate Regime. Revista de Estudos e Pesquisas Avançadas do Terceiro Setor, Brasília, DF, v. 1, n. 1, p. 69-83, 2018. https://doi.org/10.31501/repats.v1i1.9930

BARROS-PLATIAU, Ana Flávia. When emergent countries reform global governance of climate change: Brazil under Lula. Revista Brasileira de Política Internacional, Brasília, DF, v. 53, n. SPE, p. 73-90, 2010. https://doi.org/10.1590/S0034-73292010000300005

BARROS, Ana Flávia. O Brasil na governança das grandes questões ambientais contemporâneas, país emergente? Brasília: Cepal; Ipea, 2011. (Texto para Discussão, n. 40).

BEDERMAN, David J. The sea. In: FASSBENDER, Bardo et al. (ed.). The Oxford handbook of the history of international law. Oxford University Press, 2012.

BENNETT, Nathan J.; SATTERFIELD, Terre. Environmental governance: A practical framework to guide design, evaluation, and analysis. Conservation Letters, v. 11, n. 6, p. e12600, 2018. https:// doi.org/10.1111/conl.12600

BEAU, Rémi; LARRÈRE, Catherine. Penser l'anthropocène. Presses de Sciences Po, 2018. 
BEURRIER, Jean-Pierre. Droit international de L'Environment. ed. 5. Paris: Ed. A. Pedone, 2017.

BIERMANN, Frank et al. The fragmentation of global governance architectures: A framework for analysis. Global Environmental Politics, Cambridge, Mass., v. 9, n. 4, p. 14-40, 2009. https://doi. org/10.1162/glep.2009.9.4.14

BIERMANN, Frank. Planetary boundaries and earth system governance: Exploring the links. Ecological Economics, [S. l.], v. 81, p. 4-9, 2012. https://doi.org/10.1016/j.ecolecon.2012.02.016

BLASIAK, Robert et al. Corporate control and global governance of marine genetic resources. Science advances, [S. l.], v. 4, n. 6, p. eaar5237, 2018. https://doi.org/10.1126/sciadv.aar5237

BLASIAK, Robert et al. Negotiating the use of biodiversity in marine areas beyond national jurisdiction. Frontiers in Marine Science, [S. l.], v. 3, p. 224, 2016. https://doi.org/10.3389/fmars.2016.00224

BREITMEIER, Helmut; YOUNG, Oran R.; ZÜRN, Michael. Analyzing international environmental regimes: from case study to database. Cambridge: MIT Press, 2006. https://doi.org/10.7551/mitpress/1264.001.0001

BROGGIATO, Arianna et al. Mare Geneticum: Balancing Governance of Marine Genetic Resources in International Waters. The International Journal of Marine and Coastal Law, [S. l.], v. 33, n. 1, p. 3-33, 2017. https://doi.org/10.1163/15718085-13310030

BURGES, Sean W. Brazil as a bridge between old and new powers?. International Affairs, [S. l.], v. 89, n. 3, p. 577-594, 2013. https://doi.org/10.1111/1468-2346.12034

CAMARGO, Suzana. Rio de Janeiro é a primeira cidade brasileira a banir canudos plásticos. In: Conexão Planeta [ Web site] , 03 jul. 2018. Disponível em: http://conexaoplaneta.com.br/blog/rio-de-janeiro-pode-ser-primeira-cidade-brasileira-a-banir-canudos-plasticos. Acesso em: 11 fev. 2019.

CEBALLOS, Gerardo; EHRLICH, Paul R.; DIRZO, Rodolfo. Biological annihilation via the ongoing sixth mass extinction signaled by vertebrate population losses and declines. Proceedings of the National Academy of Sciences, Washington (D.C.), v. 114, n. 30, p. E6089-E6096, 2017. https://doi. org/10.1073/pnas.1704949114

DECLARAÇÃO de Belém sobre a cooperação em investigação e inovação no oceano Atlântico. Lisboa, 2017. Disponível em: https://ec.europa.eu/research/iscp/pdf/belem_statement_2017_pt.pdf. Acesso em: 11 fev 2019.

DRANKIER, Petra et al. Marine genetic resources in areas beyond national jurisdiction: access and benefit-sharing. The International Journal of Marine and Coastal Law, [S. l.], v. 27, n. 2, p. 375-433, 2012. https://doi.org/10.1163/157180812X637984

DRUEL, Elisabeth et al. A long and winding road: International discussions on the governance of marine biodiversity in areas beyond national jurisdiction. Paris: IDDRI, 2012. (IDDRI Study 07/13).

DUPUY, Pierre-Marrie; VIÑUALES, Jorge E. Introduction au droit international de l'environment. Bruxelas: Bruylant, 2015.

EU reaches agreement on single-use plastic ban. In: DW, [S. I.], 19 dez. 2018. Disponível em: https:// www.dw.com/en/eu-reaches-agreement-on-single-use-plastic-ban/a-46797494. Acesso em: 11 fev. 2019.

FREESTONE, Daniel. Can existing institutions protect biodiversity in areas beyond national jurisdiction? Experiences from two on-going processes. Marine Policy, Surrey, v. 49, p. 167-175, 2014. https://doi.org/10.1016/j.marpol.2013.12.007 
FINAL Report of the Forty-first Antarctic Treaty Consultative Meeting. Buenos Aires: ATCM, 2018. Disponível em: https://www.ats.aq/documents/ATCM41/fr/ATCM41 froo1 e.pdf. Acesso em: 11 fev. 2019.

GREEN, Jessica F.; AULD, Graeme. Unbundling the regime complex: the effects of private authority. Transnational environmental law, [S. l.], v. 6, n. 2, p. 1-26, 2016. https://doi.org/10.1017/ $\underline{\text { S2047102516000121 }}$

GOH, Evelyn; PRANTL, Jochen. Why strategic diplomacy matters for Southeast Asia. East Asia Forum, [S. l.], v. 9, n. 2, 2017.

PERSHING, Andrew J. et al. Oceans and marine resources. In: NATIONAL CLIMATE ASSESSMENT, 4., Washington DC. Proceedings [...], Washington DC : U.S. Global Change Research Program, 2018. v. 2, p. 353-390. Disponível em: https://nca2018.globalchange.gov/chapter/9/. Acesso em: 11 fev. 2019.

HARDEN-DAVIES, Harriet. The next wave of science diplomacy: marine biodiversity beyond national jurisdiction. ICES Journal of Marine Science, [S. l.], v. 75, n. 1, p. 426-434, 2017. https://doi. org/10.1093/icesjms/fsx165

HO, Peter. The Challenges Of Governance In A Complex World. World Scientific, 2017. https://doi. org/10.1142/10759

INOUE, C. Global Climate Governance: a proposed analytical framework under construction. Carta Internacional, [S. l.], v. 11, n. 1, 2016, pp. 91-117. https://doi.org/10.21530/ci.v11n1.2016.242

INTERGOVERNMENTAL Conference on Marine Biodiversity of Areas Beyond National Jurisdiction. US ; United Nations, [2019]. Disponível em: https://www.un.org/bbnj/. Acesso em: 11 fev. 2019.

INTERNATIONAL INSTITUTE FOR SUSTAINABLE DEVELOPMENT (IISD). 1st Session of the Intergovernmental Conference (IGC) on an International Legally Binding Instrument under the United Nations Convention on the Law of the Sea (UNCLOS) on the Conservation and Sustainable Use of Marine Biological Diversity of Areas Beyond National Jurisdiction (BBNJ). Nova York: IISD, 2018. Disponível em: http://enb.iisd.org/oceans/bbnj/igc1/. Acesso em: 11 fev. 2019

JOHNSON, J.; REYNOLDS, H.; MYCOFF, J. Political science research methods. [S. l.]: Cq Press, 2016. KAVALSKI, Emilian (org.). World politics at the edge of chaos: reflections on complexity and global life. New York: SUNY Press, 2016.

KERVRAN, David Dumoulin; PEPIN-LEHALLEUR, Marielle. Agir-en-réseau: modèle d'action ou catégorie d'analyse?. Rennes: Presses Universitaires de Rennes, 2012. https://doi.org/10.4000/ books.pur.70677

KEOHANE, R.; VICTOR, D. The regime complex for climate change. Perspectives on Politics, New York, v. 9, p. 7- 23, 2011. https://doi.org/10.1017/S1537592710004068

KRASNER, Stephen. Structural Causes and Regime Consequences: Regimes as Intervening Variables. International Organization, Cambridge, Mass, v. 36, n. 2, p. 185-205, 1982. https://doi.org/10.1017/ $\underline{\text { So020818300018920 }}$

LE PRESTRE, Philippe. Global Ecopolitics Revisited: towards a complex governance of global environmental problems. Nova York: Routledge, 2017. https://doi.org/10.4324/9781315563695

LUIZ, Gabriel; CASSELA, Vinícius. Ibaneis sanciona lei que proíbe canudos e copos de plástico no DF. In: G1, [S. l.], 07 fev. 2019. Disponível em: https://g1.globo.com/df/distrito-federal/noticia/2019/02/07/ ibaneis-sanciona-lei-que-proibe-canudos-e-copos-de-plastico-no-df.ghtml. Acesso em: 11 fev. 2019. 
MALJEAN-DUBOIS, Sandrine. La Convention de Rio sur la diversité biologique. In: NÉGRI, Vincent (org.). La diversité dans la gouvernance internationale: perspectives culturelles, écologiques et juridiques. Bruylant, 2016.

MAZZEGA, Pierre et al. Mapping ocean-related main issues onto decision of environmental Conferences of Parties. In: OLIVEIRA, Carina; GALINDO, George; TELES, Solange; MONTALVERNE, Tarin (org.). Meio Ambiente Marinho e Direito. 1ed. Curitiba: Juruá, 2018. v. 2, p. 03-21.

Recebido: $18 / 02 / 2019$

Aceito: 27/05/2019

\section{Biografia do autor}

\section{Igor Magri de Queiroz}

Graduando em Relações Internacionais pelo Instituto de Relações Internacionais - Universidade de Brasília (IREL/UnB). Bolsista da Coordenação de Aperfeiçoamento de Pessoal de Nível Superior (CAPES), em graduação-sanduíche na Faculté de Droit et de Science Politique - Aix-Marseille Université, projeto "A Estratégia Brasileira para a Gestão Sustentável dos Recursos Marinhos Vivos" (Projetos Estratégicos, DRI). ORCID: http://orcid.org/o000-0002-1214-6934; E-mail de contato: igormagria@gmail.com.

Instituição: Instituto de Relações Internacionais - Universidade de Brasília (IREL/UnB).

Localização: Campus Universitário Darcy Ribeiro Prédio do Ipol/Irel s/n - Asa Norte, DF, 70904-970 